\title{
Negative Beanspruchung von Schweizer Lehrpersonen. Trends von 2006 bis 2014
}

\section{Anita Sandmeier ${ }^{1}$, Doris Kunz Heim, Béat Windlin und Andreas Krause}

In diesem Beitrag wird untersucht, ob und inwieweit sich das Ausmass der negativen Beanspruchung bei Lehrpersonen in der Schweiz der 5. - 9. Schulstufe (7. - 11. HarmoS) in den letzten zehn Jahren verändert hat. Auf der Grundlage einer nationalen sequentiellen Querschnittsanalyse können erstmals Aussagen zu Trends zwischen 2006 und 2014 gemacht werden: Das Ausmass der negativen Beanspruchung bleibt bei regionalen Unterschieden insgesamt relativ stabil und die Lehrkräfte sind trotz anhaltend hohen Anforderungen mit ihrem Beruf zufrieden. Die Befunde werden vor dem Hintergrund der national bedeutsamen Bildungsreformen in diesem Zeitraum diskutiert.

\section{Einleitung}

Die Forschung zu Belastung und Beanspruchung im Lehrberuf ist seit der Jahrtausendwende ein Schwerpunkt der empirischen Lehrerforschung (Rothland, 2013). Auch in der Schweiz gibt es eine Vielzahl von kantonalen Studien, die grundlegende Erkenntnisse generiert haben (u.a. Albisser, Kirchhoff \& Albisser, 2009; Bieri, 2006; Herzog, 2007; Keller-Schneider, 2010; Nido, 2012; Trachsler et al., 2006). Da die Studien in Abhängigkeit von der Ausrichtung der Forschungsgruppe unterschiedliche theoretische Fundierungen und empirische Zugänge haben, war bislang ein Vergleich der Befunde über die Regionen hinweg und Aussagen zu Trends der Beanspruchung der Schweizer Lehrpersonen kaum möglich. Im Rahmen der nationalen Studie «Health Behavior in School-Aged Children» $[\mathrm{HBSC}]^{2}$ wurde über mehrere Erhebungszeitpunkte dasselbe Erhebungsinstrument in sprachregional übergreifenden Stichproben eingesetzt, was erstmals die Gelegenheit bietet, die negative Beanspruchung von Lehrpersonen in der ganzen Schweiz über mehrere Jahre hinweg vergleichend zu analysieren und regionale Entwicklungen zu betrachten. Als Ausgangspunkt der Erwartungen und Grundlage für die Interpretation der Ergebnisse werden die bildungspolitischen Reformen in der Schweiz herangezogen. 


\section{Theoretischer Hintergrund und Forschungsstand}

Die der RBSL-Studie zugrundeliegenden theoretischen Annahmen basieren auf dem Rahmenmodell der Belastung und Beanspruchung, das äussere Einflussfaktoren (Belastungen) von Reaktionen der arbeitenden Person (psychische Beanspruchung) unterscheidet (Ulich \& Wülser, 2015). Rudow (2000) formuliert dies für das Arbeitsfeld Schule folgendermassen: «Unter Belastung sind alle diejenigen körperlichen, geistigen und sozialen Anforderungen in der pädagogischen Tätigkeit zu verstehen, die unabhängig vom Individuum existieren und potentiell Beanspruchungen hervorrufen» (ebd., S. 36). Dabei sind Belastungen neutral konnotiert, d.h. sie sind nicht per se negativ, sondern führen zu einer Reaktion der Person (Beanspruchung), die sowohl positiv als auch negativ sein kann. Die Rahmenkonzeption von Rudow (1994) geht davon aus, dass die objektiven Belastungsfaktoren (Merkmale der Arbeitsaufgabe und die Arbeitsbedingungen) durch subjektive Prozesse wie Wahrnehmung, Bewertung und kognitive Verarbeitung in subjektive Belastung münden. Dabei sind die subjektiven Prozesse abhängig von den Handlungsvoraussetzungen des Individuums. Aus der Belastung resultieren kurzfristig Beanspruchungsreaktionen, die zeitlich begrenzt, zielbezogen und im Handlungskontext auftreten (positiv z.B. als kognitive Aktivierung, Glück oder Flow-Erleben, negativ z.B. als psychische Ermüdung, Monotonie oder Stress). Von dieser kurzfristigen, reversiblen Beanspruchungsreaktion wird die langfristige Beanspruchungsfolge unterschieden, welche ein unabhängig vom Handlungskontext überdauerndes, motivbezogenes, bedingt reversibles Phänomen ist (positiv als Gefühl der Kompetenz, des Engagements und der Arbeitszufriedenheit, negativ als Überforderungsgefühle, Beeinträchtigung der Gesundheit und Burnout). Neben diesem grundlegenden Rahmenmodell werden in der Lehrerforschung zunehmend Modelle der Arbeits- und Organisationspsychologie herangezogen, um den Prozess der Beanspruchung zu analysieren. Das Effort-Reward-Imbalance Modell (Siegrist, 1996), das davon ausgeht, dass negative Beanspruchung durch Gratifikationskrisen entsteht, wurde u.a. von Lehr, Hillert und Keller (2013) an einer Lehrerstichprobe getestet. Das Job-Demands-Resources Modell (Hakanen, Bakker \& Schaufeli, 2006) postuliert, dass negative Beanspruchung dann entsteht, wenn das Individuum zu wenig Ressourcen hat, um die beruflichen Anforderungen zu bewältigen. Der im theoretischen Modell aufgezeigte Erschöpfungsprozess wurde von Baeriswyl, Krause und Kunz Heim (2014) für Lehrpersonen bestätigt.

Obwohl Belastung und Beanspruchung seit längerer Zeit ein Schwerpunkt der deutschsprachigen empirischen Lehrerforschung ist, stellt sich die Befundlage widersprüchlich und unbefriedigend dar und es besteht ein Missverhältnis zwischen der grossen Zahl an Studien und dem geringen Erkenntnisfortschritt (Rothland, 2013). Als relativ gesichert kann gelten, dass Disziplinprobleme, Heterogenität der Schulklasse, bzw. unterschiedliche Lernvoraussetzungen der Schülerinnen und Schüler, Zeitdruck und der Umgang mit Reformen und 
Neuerungen im Schulsystem von vielen Lehrkräften als Belastungen wahrgenommen werden (Krause, Dorsemagen \& Baeriswyl, 2013). Ebenfalls stabil ist der Befund, dass Lehrpersonen zwar hohen Belastungen ausgesetzt sind, jedoch eine hohe Arbeitszufriedenheit aufweisen (Bieri, 2006; Schult, Münzer-Schrobildgen \& Sparfeldt, 2015). Der Zusammenhang mit beruflichen Bewältigungsmustern wurde von Schaarschmidt \& Fischer (2001) erstmals aufgezeigt und wiederholt repliziert (u.a. Albisser, Kirchhoff, \& Albisser, 2009; Klusmann, Kunter, Trautwein, \& Baumert, 2006; Nido, 2012). Will man jedoch die Beanspruchung von Lehrkräften über eine längere Zeit hinweg im Sinne eines Monitorings überblicken, ist die Datenlage im deutschsprachigen Raum relativ dünn. Schaarschmidt und Kieschke (2013) konstatieren in ihrem Überblicksartikel jedoch dezidiert: «(...) unbestritten dürfte sein, dass sich in den letzten Jahren noch eine weitere Zuspitzung der Belastungssituation abzeichnet» (S. 82). Den Grund dafür sehen sie in der stetigen Zunahme der beruflichen Aufgaben bei gleichzeitiger Verschlechterung der Bedingungen (ebd.).

In der Schweiz besteht eine Vielzahl an kantonalen Untersuchungen zu Belastung und Beanspruchung mit unterschiedlichen Forschungszugängen: Studien mit einem qualitativen Ansatz betrachten Belastung und Beanspruchung in einem berufsbiographischen Zusammenhang, z.B. die Erfahrungen von Lehrpersonen, welche wegen Erschöpfungssymptomen längere Zeit vom Unterricht dispensiert waren (Hangartner, Hofer \& Freisler, 2012) oder die Beanspruchungs- und Bewältigungsprozesse von Lehrpersonen, die im Beruf bleiben im Vergleich zu Lehrpersonen, welche den Beruf ganz oder zeitweise verlassen (Herzog, 2007). Hangartner et al. (2012) stellten dabei fest, dass ein Grossteil der Lehrpersonen, die erschöpfungsbedingt krankgeschrieben wurden, Reformen und damit zusammenhängende Veränderungen der Bedingungen ihres Unterrichts als Belastung wahrgenommen hatten. Am häufigsten sind quantitative schriftliche Befragungen, welche verschiedenste Aspekte von selbstreferentiellen Auskünften zu Belastung und Beanspruchung im Querschnitt in regionalen Stichproben untersuchen. Die Frage, wie sich die negative Beanspruchung der berufstätigen Schweizer Lehrpersonen über die Jahre im Trend entwickelt, ist dabei schwierig zu beantworten, da die Studien auf verschiedenen Stichproben beruhen (unterschiedliche untersuchte Schulstufen, unterschiedliche Regionen) und unterschiedliche Instrumente einsetzen, um negative Beanspruchung zu messen. Diese reichen von der auf einer Clusteranalyse basierenden Erfassung von Arbeitsbezogenen Verhaltens- und Erlebensmuster von Schaarschmidt und Fischer (1997), eingesetzt u.a. von Nido (2012) und Albisser, Kirchhoff und Albisser (2009), über das Beanspruchungsscreening (Hacker \& Reinhold, 1999), eingesetzt u.a. von Trachsler et al. (2006) und Ulich, Inversini und Wülser (2002), der Skala zu beruflicher Belastung von Enzmann und Kleiber (1989), eingesetzt u.a. von Windlin, Kuntsche und Delgrande (2011), Delgrande, Kuntsche und Sidler (2005) und Forneck und Schriever (2001), bis hin zu klassischen Burnout-Inventaren wie dem Maslach Burnout-Inventory (Maslach \& Jackson, 
1981), eingesetzt u.a. von Grunder und Bieri (1995) und Forneck und Schriever (2001) und dem Copenhagen Burnout-Inventory (Kristensen, Borritz, Villadsen \& Christensen, 2005), eingesetzt u.a. von Kunz Heim, Sandmeier und Krause (2014a) und Baeriswyl, Krause und Kunz Heim (2014).

Längsschnittliche Studien zu Beanspruchungsfolgen sind selten und zeitlich beschränkt. Albisser et al. (2009) stellten im Kanton Zürich eine hohe interindividuelle Stabilität über zwei Jahre fest. Erhöhungen der negativen Beanspruchung hingen mit fehlender Zielkontrolle zusammen, d.h. wenn die Erreichung von beruflichen Zielen aufgrund äusserer Umstände und ungenügender eigenen Ressourcen nicht mehr realisierbar erschienen. Trachsler et al. (2006) haben im Kanton Thurgau die Umsetzung von Reformen und deren Auswirkung auf die Beanspruchung während zwei Jahren längsschnittlich evaluativ begleitet. Die Lehrkräfte gaben in den Interviews an, dass das Reformtempo und die Menge an Entwicklungsprojekten sie an die Grenze ihrer Belastbarkeit bringen. In den quantitativen Daten konnte jedoch keine Zunahme der negativen Beanspruchung festgestellt werden.

So vielfältig die Forschung in diesem Bereich in der Schweiz ist, so schwer fällt es, hinter den vielen punktuellen Befunden ein Gesamtbild zur Beanspruchungssituation des Lehrpersonals zu erkennen. Die kantonalen Resultate können aufgrund der unterschiedlich zusammengesetzten Stichproben und der divergierenden Erhebungsinstrumente nicht miteinander verglichen werden, weshalb es bis dato nur beschränkt möglich war Aussagen zu machen zu regionalen Unterschieden in der Beanspruchung oder zu Trends über die Zeit.

\section{Belastung und Beanspruchung über einen Längeren Zeitraum auf nationaler Ebene}

Eine Quelle für Aussagen zur Beanspruchungssituation der Lehrerinnen und Lehrer sind die Mitgliederbefragungen des Dachverbands Schweizer Lehrerinnen und Lehrer $[\mathrm{LCH}]$, die alle vier Jahre durchgeführt werden. Diese zeigen, dass die zeitliche Belastung und die geleisteten Überstunden zugenommen haben zwischen 1999 und 2009 (Landert \& Brägger, 2009). Die berufliche Zufriedenheit andererseits hat sich zwischen 2001 und 2014 «unübersehbar» verbessert (Landert, 2014). Neben den erwähnten Befragungen des LCH, deren Aussagen rein deskriptiv sind und sich auf die Deutschschweiz beschränken, haben Delgrande, Kuntsche und Sidler (2005) erstmals eine regional übergreifende Stichprobe von Schweizer Lehrkräften des 5. bis 9. Schuljahres untersucht. Sie konnten die Skalen der Arbeitsüberforderung und -unzufriedenheit von Enzmann und Kleiber (1989) validieren und als je separate Dimension identifizieren. Die Autoren fanden positive Zusammenhänge zwischen diesen beiden Skalen und depressiven und somatischen Beschwerden. Ausserdem zeigte sich, dass bei den Westschweizer Lehrpersonen Arbeitsüberforderung und depressive 
Beschwerden weiterverbreitet sind als in der Deutschschweiz (Delgrande et al., 2005). In den Jahren 2006³, 2010 und 2014 wurden dieselben Skalen erneut in einer gesamtschweizerischen Stichprobe eingesetzt. Diese replikativen Querschnittstudien bilden die Grundlage für die hier präsentierten Analysen. Aufgrund dieser Daten ist es erstmals möglich, Trends bezüglich negativer Beanspruchungsfolgen von Schweizer Lehrpersonen zu untersuchen. Entsprechende Studien zu Trends in der negativen Beanspruchung des Lehrpersonals gibt es im deutschen Sprachraum zu anderen nationalen Bildungssystemen nach unserem Kenntnisstand keine.

\section{Fragestellung und Hypothesen}

Der vorliegende Beitrag fragt danach, ob sich die Ausprägung der negativen Beanspruchungsfolgen der Schweizer Lehrpersonen der 5. - 9. Schulstufe zwischen den Erhebungsjahren 2006, 2010 und 2014 und zwischen verschiedenen Regionen voneinander unterscheidet und ob sich bestimmte Trends der Veränderung beobachten lassen.

Hypothesen zu Trends zu formulieren, die für die gesamte Gruppe von Schweizer Volksschullehrkräften gelten, ist anspruchsvoll, da Beanspruchungsprozesse abhängig von zahlreichen individuell und kontextuell ausprägten Faktoren sind. Die dezentrale Steuerung des Schweizer Bildungssystems führt dazu, dass sich die kontextuellen Bedingungen lokal stark unterscheiden. Die Tatsache, dass Reformen von Lehrpersonen in zahlreichen Studien als Belastungsfaktor genannt werden (Albisser et al., 2009; Nido, 2012; Trachsler et al., 2006), lenkt den Blick auf zwei national bedeutsame bildungspolitische Entwicklungen zwischen 2006 und 2014: Seit der Annahme des Bildungsrahmenartikels im Jahr 2006 wurden schweizweit bildungspolitische Reformen angestossen, die alle Kantone betreffen, u.a. die Vereinheitlichung der Dauer der Bildungsstufen, die Festlegung von nationalen Bildungsstandards und die Einführung von zwei Fremdsprachen auf der Primarstufe (Criblez, Müller \& Oelkers, 2011). Diese Reformen wurden in den meisten Kantonen zwischen 2006 und 2015 umgesetzt (EDK, 2015). Die ebenfalls im Bildungsrahmenartikel vorgesehene sprachregionale Harmonisierung der Lehrpläne wurde mit der Implementation des «Plan d'études romand» in allen Westschweizer Kantonen von 2010 bis 2014 umgesetzt (CIIP, 2015). Die Einführung des Deutschschweizer Pendants ist weniger weit fortgeschritten, der «Lehrplan 21» liegt seit 2014 vor und dessen Implementierung wird in aktuell zehn Kantonen vorbereitet (D-EDK, 2015). Zusätzlich zu den Massnahmen rund um den Bildungsrahmenartikel führte auf nationaler Ebene ab 2004 das Behindertengleichstellungsgesetz und die Neugestaltung der Aufgabenteilung zwischen Bund und Kantonen [NFA] zu tiefgreifenden Reformen im sonderpädagogischen Bereich (EDK, 2007; Moser Opitz, 2011). Die verstärkte integrative Schulung von Kindern mit besonderem 
Bildungsbedarf führte zu einer höheren Heterogenität innerhalb der Schulklasse, die als zentraler Belastungsfaktor von Lehrkräften identifiziert wurde (u.a. Trachsler et al., 2006).

Vor dem Hintergrund dieser national bedeutsamen Bildungsreformen mit teilweise weitreichenden Auswirkungen auf den Unterrichtsalltag erwarten wir, dass das Ausmass der negativen Beanspruchung im Trend zwischen 2006 und 2014 steigt.

\section{Methode}

\section{Studien- und Stichprobenbeschreibung}

Im Rahmen der internationalen, unter der Ägide der WHO durchgeführten Studie "Health Behaviour in School-Aged Children» [HBSC] führte Sucht Schweiz (ehemals Schweizerische Fachstelle für Alkohol- und andere Drogenprobleme) in den Jahren 2002, 2006, 2010 und 2014 neben der Schülerbefragung auch eine standardisierte schriftliche Befragung der Lehrpersonen durch ${ }^{4}$. Die Befragungen fanden jeweils zwischen Januar und April statt. In den ersten beiden Befragungen $(2002,2006)$ wurden Arbeitsüberforderung, Arbeitsunzufriedenheit, sowie depressive und somatische Beschwerden als Indikatoren von negativen Beanspruchungsfolgen von Lehrkräften erhoben. Ab 2010 wurden zusätzlich mehrere Skalen zu Belastungen, personalen und sozialen Ressourcen und weitere Indikatoren für negative und positive Beanspruchungsfolgen erhoben, um Belastungs- und Beanspruchungsprozesse detaillierter analysieren zu können (Baeriswyl, Krause \& Kunz Heim, 2014; Kunz Heim, Sandmeier \& Krause, 2014a, 2014b).

Im Rahmen dieses Beitrags werden die Daten zu Arbeitsüberforderung, Arbeitsunzufriedenheit, depressive und somatische Beschwerden in den Erhebungsjahren 2006, 2010 und 2014 verglichen. Die Daten der ersten Erhebung (2002) werden nicht mit einbezogen, da die Lehrkräfte des Kantons Waadt in diesem Erhebungsjahr nicht zu diesem Thema befragt werden konnten.

Die Klassen und Lehrpersonen wurden durch eine nationale Zufallsstichprobe ausgewählt auf der Grundlage des Verzeichnisses aller Klassen des 5. bis 9 . Schuljahres der öffentlichen Schulen des Bundesamtes für Statistik (siehe auch Windlin, Kuntsche \& Delgrande, 2011). Durch dieses Vorgehen wurde eine für die Schweiz repräsentative Stichprobe von Schulklassen gewonnen. Die Stichprobe der Lehrpersonen ist eingeschränkt repräsentativ, da pro ausgewählter Klasse nur jene Lehrkraft befragt wurde, die die Klassenbefragung durchführte. Dies waren in der Regel Klassenlehrpersonen. Fachlehrkräfte sind deutlich untervertreten, ebenso Lehrkräfte, die kleine Pensen unterrichten. Bei den einzelnen Befragungen handelt es sich jeweils um Querschnittstudien, die Zufallsstichprobe wurde für jede Studie neu gezogen, d.h. die Lehrpersonen nahmen im Normalfall nur einmal an der Studie teil. 
Um eine Konfundierung aufgrund der unterschiedlichen Zusammensetzung der Kohorten zu kontrollieren, werden diese in einem ersten Schritt bezüglich relevanter Merkmale miteinander verglichen (vgl. Tabelle 1).

Tabelle 1: Fallzablen nach Erhebungszeitpunkt, Analysegruppen und weiteren relevanten Faktoren

\begin{tabular}{|c|c|c|c|c|c|c|c|c|c|}
\hline \multicolumn{10}{|c|}{ Erhebungszeitpunkt } \\
\hline \multirow{3}{*}{$\begin{array}{l}\text { Teilnahme- } \\
\text { quote }\end{array}$} & \multicolumn{2}{|c|}{2006} & \multicolumn{2}{|c|}{2010} & \multicolumn{2}{|c|}{2014} & \multicolumn{2}{|c|}{ Total } & \multirow{3}{*}{$\begin{array}{l}\text { Unterschiede der } \\
\text { Faktoren nach } \\
\text { Erhebungsjahr } \\
\text { Chi2 (df,N) }\end{array}$} \\
\hline & \multicolumn{2}{|c|}{$82.6 \%$} & \multicolumn{2}{|c|}{$82.1 \%$} & \multicolumn{2}{|c|}{$78.5 \%$} & \multirow[b]{2}{*}{$\mathbf{N}$} & \multirow[b]{2}{*}{$\%$} & \\
\hline & $\mathbf{N}$ & $\%$ & $\mathbf{N}$ & $\%$ & $\mathbf{N}$ & $\%$ & & & \\
\hline $\begin{array}{l}\text { Gesamtstich- } \\
\text { probe }\end{array}$ & 566 & 100 & 585 & 100 & 567 & 100 & 1718 & 100 & \\
\hline \multicolumn{10}{|l|}{ Region } \\
\hline $\mathrm{D}$ & 407 & 71.9 & 420 & 71.8 & 400 & 70.5 & 1227 & 71.4 & \multirow{3}{*}{$\begin{array}{l}\mathrm{Chi}^{2} \\
(4,1718)=3.77 \\
\mathrm{p}=.439\end{array}$} \\
\hline $\mathrm{F}$ & 126 & 22.3 & 141 & 24.1 & 144 & 25.4 & 411 & 23.9 & \\
\hline I & 33 & 5.8 & 24 & 4.1 & 23 & 4.1 & 80 & 4.7 & \\
\hline \multirow{3}{*}{$\begin{array}{l}\text { Geschlecht } \\
\text { männlich } \\
\text { weiblich }\end{array}$} & & & & & & & & & \multirow{3}{*}{$\begin{array}{l}\mathrm{Chi}^{2} \\
(2,1718)=5.83 \\
\mathrm{p}=.054\end{array}$} \\
\hline & 340 & 60.1 & 324 & 55.4 & 301 & 53.1 & 965 & 56.2 & \\
\hline & 226 & 39.9 & 261 & 44.6 & 266 & 46.9 & 753 & 43.8 & \\
\hline \multicolumn{10}{|l|}{ Klassenstufe } \\
\hline 5./6. Jahr & 223 & 39.4 & 210 & 35.9 & 196 & 34.6 & 629 & 36.6 & \multirow{3}{*}{$\begin{array}{l}\mathrm{Chi}^{2} \\
(4,1718)=3.95 \\
\mathrm{p}=.412\end{array}$} \\
\hline 7./8. Jahr & 220 & 38.9 & 242 & 41.4 & 228 & 40.2 & 690 & 40.2 & \\
\hline 9. Jahr & 123 & 21.7 & 133 & 22.6 & 143 & 25.2 & 399 & 23.2 & \\
\hline \multicolumn{9}{|l|}{$\begin{array}{l}\text { Berufs- } \\
\text { erfahrung }\end{array}$} & \multirow{4}{*}{$\begin{array}{l}\mathrm{Chi}^{2} \\
(4,1718)=1.12 \\
\mathrm{p}=.892\end{array}$} \\
\hline$\leq 5$ Jahre & 109 & 19.3 & 120 & 20.5 & 113 & 19.9 & 342 & 19.9 & \\
\hline 6 - 25 Jahre & 285 & 50.4 & 294 & 50.3 & 296 & 52.2 & 875 & 50.9 & \\
\hline$>25$ Jahre & 172 & 30.4 & 171 & 29.2 & 158 & 27.9 & 501 & 29.2 & \\
\hline \multicolumn{9}{|l|}{ Pensum } & \multirow{4}{*}{$\begin{array}{l}\mathrm{Chi}^{2} \\
(2,1151)=3.78 \\
p=.151^{5}\end{array}$} \\
\hline$\leq 10 \mathrm{Lek} / \mathrm{W}$ & 158 & 28.1 & 9 & 1.5 & 11 & 1.9 & 178 & 10.4 & \\
\hline 11-20 Lek/W & 193 & 34.3 & 85 & 14.5 & 105 & 18.5 & 383 & 22.3 & \\
\hline > $20 \mathrm{Lek} / \mathrm{W}$ & 212 & 37.7 & 491 & 83.9 & 450 & 79.4 & 1153 & 67.1 & \\
\hline
\end{tabular}

In den für die Analyse relevanten drei Erhebungsjahren wurden jene Lehrpersonen ausgeschlossen, zu denen Angaben zu Geschlecht, Klassenstufe und Berufserfahrung fehlen. Aufgrund dieser Ausschlüsse können die Ergebnisse dieser Analyse vereinzelt leicht abweichen von weiteren publizierten Ergeb- 
nissen aus Daten der erwähnten Erhebungsjahre. Nach diesen Bereinigungen resultiert eine Stichprobe von 1718 Lehrpersonen (vgl. Tabelle 1). Bezüglich der Zusammensetzung nach Region, Klassenstufe, Berufserfahrung, Geschlecht und Pensum ${ }^{5}$ unterscheiden sich die Stichproben der drei Erhebungsjahre nicht signifikant.

\section{Operational is ierung}

Die Westschweiz und das Tessin wurden aufgrund statistischer Rahmenbedingungen (zu geringe Stichprobengrösse im Tessin) in einer Kategorie zusammengefasst. Dadurch resultiert die Variable "Region» (1=Deutschschweiz, $2=$ Westschweiz und Tessin)

Die Skalen Arbeitsüberforderung und Arbeitsunzufriedenheit von Enzmann und Kleiber (1989) erfassen die negative Beanspruchung in der konkreten beruflichen Tätigkeit ${ }^{6}$. Die Dimension Arbeitsüberforderung enthält Aussagen zu Gefühlen der allgemeinen Überforderung, des Nicht-Abschalten-Könnens und der Beanspruchung durch die Verantwortung für die Schülerinnen und Schüler. Die Skala verfügt zu allen drei Messzeitpunkten und in beiden Regionen über eine gute Reliabilität (Cronbach's a $=.768$ bis .80 ). Arbeitsunzufriedenheit wird erhoben als generalisierte Einstellung zum Arbeitsverhältnis. Eine Eigenschaft dieser Skala besteht darin, dass einzelne Items invertiert formuliert sind, z.B. «Mein Beruf macht mir Spass». Die Skala misst die allgemeine Bewertung des Berufes, das Befinden am Arbeitsplatz und die Möglichkeit zur Selbstentfaltung. Beide Skalen wurden mit einem fünfstufigen Antwortformat erhoben und verfügen zu allen drei Messzeitpunkten und in beiden Regionen über eine gute Reliabilität (Cronbach's a $=.718$ bis .789). Gesundheitliche Beanspruchung wurde als Index depressiver und somatischer Beschwerden (Delgrande Jordan et al., 2005) erhoben. Der Abschnitt wird eingeleitet mit der Frage «Wie oft hatten Sie in den vergangenen 6 Monaten die folgenden Beschwerden?» gefolgt von folgenden Beschwerden: Kopfschmerzen, Bauchschmerzen, Rückenschmerzen, Schwindelanfälle (somatische Beschwerden) und traurig/bedrückt, gereizt/ schlecht gelaunt, nervös, müde und ängstlich/besorgt (depressive Beschwerden) ${ }^{7}$.

\section{Analysen}

Für den aktuellsten Messzeitpunkt (2014) werden deskriptive Werte berichtet, differenziert nach Geschlecht und Region. Die Frage der Veränderung zwischen den Jahren 2006, 2010 und 2014 wird mit einer mehrfaktoriellen Varianzanalyse beantwortet, in welche neben dem Erhebungszeitpunkt das Geschlecht und die Region und deren paarweise Interaktionsterme als Faktoren in die Analyse eingehen. 


\section{Ergebnisse}

Negative Beanspruchung im Jahr 2014: Arbeitsüberforderung, Arbeitsunzufriedenheit und gesundheitliche Beschwerden

Die Arbeitsüberforderung im Jahr 2014 weist im Durchschnitt ein mittleres Niveau auf $(\mathrm{M}=2.31, \mathrm{SD}=.68)$. Wenn man die Antworten auf die einzelnen Items der Skala betrachtet, bekommt man einen klareren Eindruck: $21.4 \%$ der Befragten finden, dass man im Lehrberuf ständig überfordert wird, 26.7\% haben selten das Gefühl, einmal richtig abschalten zu können und $20.5 \%$ finden den Zeitdruck zu gross, unter dem sie arbeiten ${ }^{8}$. Die Arbeitsunzufriedenheit ist leicht tiefer $(\mathrm{M}=2.03, \mathrm{SD}=.59)$ : 93.3\% der Lehrkräfte macht der Beruf Spass und 73.7\% sind mit ihrer Arbeit «eigentlich rundum zufrieden». Depressive Beschwerden $(\mathrm{M}=2.10, \mathrm{SD}=.71)$ kommen häufiger vor als somatische Beschwerden $(\mathrm{M}=1.65, \mathrm{SD}=.57)$. Die häufigste depressive Beschwerde ist Müdigkeit, $67.4 \%$ der Lehrkräfte fühlen sich mindestens einmal pro Woche 9 müde, jede vierte hat mindestens einmal pro Woche Schlafprobleme. Die häufigste somatische Beschwerde sind Rückenschmerzen, fast jede dritte Lehrperson leidet mindestens einmal pro Woche darunter.

Negative Beanspruchung 2006 bis 2014: Trends für Arbeitsüberforderung, Arbeitsunzufriedenheit und gesundheitliche Beschwerden

Was die Unterschiede zwischen den Erhebungsjahren betrifft, sind die Ergebnisse der mehrfaktoriellen Varianzanalyse in Tabelle 2 enthalten. Zur Verdeutlichung der Unterschiede sind in den Abbildungen 1 bis 4 die Mittelwerte nach Erhebungsjahr und Region grafisch dargestellt.

Die Arbeitsüberforderung der Lehrpersonen in der Gesamtgruppe unterscheidet sich nicht zwischen den drei Erhebungsjahren (vgl. Tabelle 2). Es zeigen sich jedoch regionale Unterschiede: Während in der Deutschschweiz die Arbeitsüberforderung von 2006 bis 2014 gleich hoch bleibt, sinkt sie in der Westschweiz und im Tessin zwischen 2006 und 2010 und steigt zwischen 2010 und 2014 erneut an (vgl. Abbildung 1). Zusätzlich berichten in der Westschweiz und im Tessin die Frauen eine höhere Arbeitsüberforderung als die Männer, während sich in der Deutschschweiz die Geschlechter nicht voneinander unterscheiden. 
Tabelle 2: Varianzanalysen

\begin{tabular}{|c|c|c|c|c|c|c|c|c|c|c|}
\hline & & & & \multicolumn{7}{|c|}{ Varianzanalyse } \\
\hline & \multirow[b]{2}{*}{$\begin{array}{c}2006 \\
(\mathrm{~N}=545 \\
-564)\end{array}$} & \multirow[b]{2}{*}{$\begin{array}{c}2010 \\
(\mathrm{~N}=567 \\
-572)\end{array}$} & \multirow[b]{2}{*}{$\begin{array}{c}2014 \\
(\mathrm{~N}=553 \\
-559)\end{array}$} & \multirow[b]{2}{*}{ Jahr } & \multirow[b]{2}{*}{ Region } & \multirow[b]{2}{*}{$\begin{array}{c}\text { Ge- } \\
\text { schlecht }\end{array}$} & \multicolumn{3}{|c|}{ Interaktionsterme } & \multirow[b]{2}{*}{$\begin{array}{c}\text { Ge- } \\
\text { samt }\end{array}$} \\
\hline & & & & & & & $\begin{array}{c}\text { Jahr* } \\
\text { Region }\end{array}$ & $\begin{array}{c}\text { Jahr* } \\
\text { Ge- } \\
\text { schlecht }\end{array}$ & $\begin{array}{c}\text { Region* } \\
\text { Ge- } \\
\text { schlecht }\end{array}$ & \\
\hline & $\mathrm{M}(\mathrm{SD})$ & $\mathrm{M}(\mathrm{SD})$ & $\mathrm{M}(\mathrm{SD})$ & $\begin{array}{c}\mathrm{F} \\
\mathrm{p} \\
\text { eta }^{2}\end{array}$ & $\begin{array}{c}\mathrm{F} \\
\mathrm{p} \\
\mathrm{eta}^{2}\end{array}$ & $\begin{array}{c}\mathrm{F} \\
\mathrm{p} \\
\text { eta }^{2}\end{array}$ & $\begin{array}{c}\mathrm{F} \\
\mathrm{p} \\
\mathrm{eta}^{2}\end{array}$ & $\begin{array}{c}\mathrm{F} \\
\mathrm{p} \\
\mathrm{eta}^{2}\end{array}$ & $\begin{array}{c}\mathrm{F} \\
\mathrm{p} \\
\mathrm{eta}^{2}\end{array}$ & $\begin{array}{c}\mathrm{F} \\
\mathrm{p} \\
\mathrm{R}^{2}\end{array}$ \\
\hline $\begin{array}{l}\text { Arbeitsüber- } \\
\text { forderung }\end{array}$ & $\begin{array}{l}2.29 \\
(.71)\end{array}$ & $\begin{array}{l}2.29 \\
(.66)\end{array}$ & $\begin{array}{l}2.31 \\
(.68)\end{array}$ & ns & $\begin{array}{l}49.29 \\
* * * \\
.029\end{array}$ & $\begin{array}{c}7.82 \\
* * \\
.005\end{array}$ & $\begin{array}{c}14.43 \\
* * * \\
.017\end{array}$ & ns & $\begin{array}{c}11.28 \\
* * \\
.007\end{array}$ & $\begin{array}{l}10.43 \\
* * * \\
.053\end{array}$ \\
\hline $\begin{array}{l}\text { Arbeitsunzu- } \\
\text { friedenheit }\end{array}$ & $\begin{array}{l}2.32 \\
(.45)^{\mathrm{a}}\end{array}$ & $\begin{array}{l}2.17 \\
(.60) \mathrm{b}\end{array}$ & $\begin{array}{l}2.03 \\
(.59)^{\mathrm{c}}\end{array}$ & $\begin{array}{l}20.98 \\
* * * \\
.024\end{array}$ & $\begin{array}{c}10.55 \\
* * * \\
.006\end{array}$ & ns & $\begin{array}{l}6.11 \\
* * \\
.007\end{array}$ & $\mathrm{~ns}$ & ns & $\begin{array}{l}12.29 \\
* * * \\
.062\end{array}$ \\
\hline $\begin{array}{l}\text { Depressive } \\
\text { Beschwerden }\end{array}$ & $\begin{array}{l}2.11 \\
(.68)\end{array}$ & $\begin{array}{l}2.20 \\
(.77)\end{array}$ & $\begin{array}{l}2.10 \\
(.71)\end{array}$ & ns & $\begin{array}{c}24.61 \\
* * * \\
.015\end{array}$ & $\begin{array}{c}13.91 \\
* * * \\
.008\end{array}$ & $\mathrm{~ns}$ & ns & ns & $\begin{array}{c}6.03 \\
{ }_{* * *} \\
0.032 \\
\end{array}$ \\
\hline $\begin{array}{l}\text { Somatische } \\
\text { Beschwerden }\end{array}$ & $\begin{array}{l}1.49 \\
(.52)^{\mathrm{a}}\end{array}$ & $\begin{array}{l}1.57 \\
(.57)^{\mathrm{a}}\end{array}$ & $\begin{array}{l}1.65 \\
(.57)^{b}\end{array}$ & $\begin{array}{l}8.55 \\
* * * \\
.010\end{array}$ & $\begin{array}{l}8.28 \\
* * \\
.005\end{array}$ & $\begin{array}{c}41.76 \\
* * * \\
.024\end{array}$ & ns & ns & ns & $\begin{array}{l}9.81 \\
* * * \\
.050\end{array}$ \\
\hline
\end{tabular}

Anmerkungen:

$\mathrm{M}=$ Mittelwert, $\mathrm{SD}=$ Standardabweichung, $\mathrm{N}=$ Anzahl Lehrpersonen, $\mathrm{F}=\mathrm{F}-\mathrm{Wert}$ der Varianzanalyse, $\mathrm{p}=$ Signifikanz $\left({ }^{*} \mathrm{p}<.05,{ }^{* *} \mathrm{p}<.01,{ }^{* * *} \mathrm{p}<.001\right)$, eta ${ }^{2}=$ partielles Eta-Quadrat, $\mathrm{R}^{2}=$ aufgeklärte Varianz

Die Jahre mit unterschiedlichen Kennbuchstaben $(\mathrm{a}, \mathrm{b}$ c $)$ unterscheiden sich signifikant auf dem 5\%-Niveau (Post-Hoc-Test: Scheffé).

Die Arbeitsunzufriedenheit ist in der Deutschschweiz in jedem Erhebungsjahr signifikant tiefer als in den vorherigen Jahren, d.h. sie nimmt zwischen 2006 und 2014 kontinuierlich ab. In der Westschweiz und im Tessin gibt es keine signifikanten Unterschiede zwischen den Erhebungsjahren (vgl. Tabelle 2 und Abbildung 2).

Die depressiven Beschwerden unterscheiden sich insgesamt nicht zwischen 2006, 2010 und 2014, jedoch zwischen den Regionen (vgl. Abbildung 3) und den Geschlechtern (vgl. Tabelle 2): Deutlich am häufigsten leiden weibliche Lehrkräfte aus der Westschweiz und dem Tessin unter diesen Beschwerden. Das Ausmass der somatischen Beschwerden ist 2014 in der Gesamtgruppe höher als in den beiden vorherigen Erhebungsjahren. In der Westschweiz und im Tessin sind die Beschwerden häufiger als in der Deutschschweiz (vgl. Abbildung 4), Frauen in der ganzen Schweiz berichten mehr somatische Beschwerden als Männer. 
5.00

4.00

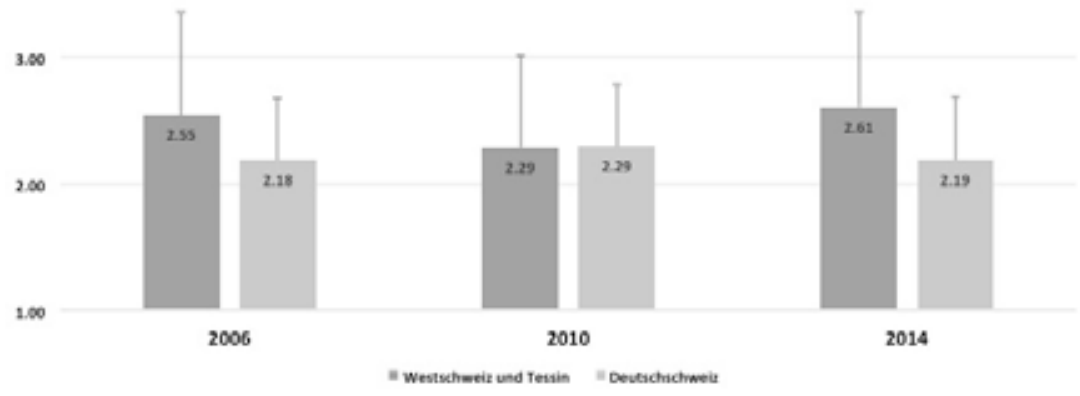

Abbildung 1: Trend Arbeitsüberforderung nach Region

Arbeitsunzufriedenheit $2006-2014$

5.00

4.90

3.00
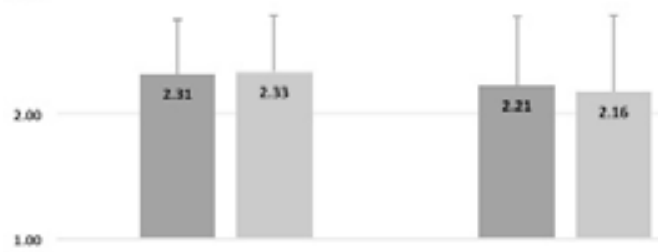

2010

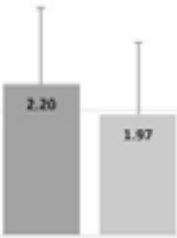

2014

" Westschmeiz und Tersin "Deutuchichneis

Abbildung 2: Trend Arbeitsunzufriedenheit nach Region 
$\$ .00$

$4 \infty$

$3 . \infty$

2.00

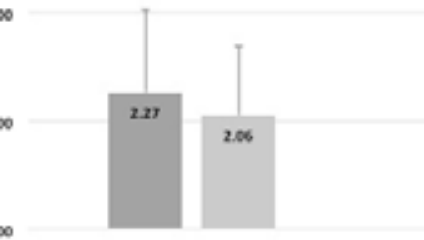

2006

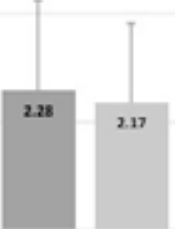

2010

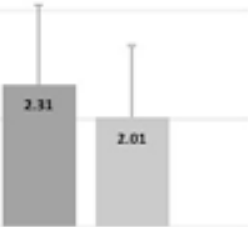

2014

"Westschmeir und Tenis "Deutuchichweis

Abbildung 3: Trend depressive Beschwerden nach Region

\section{Somatische Beschwerden 2006 - 2014}

5.00

4.00

3.00

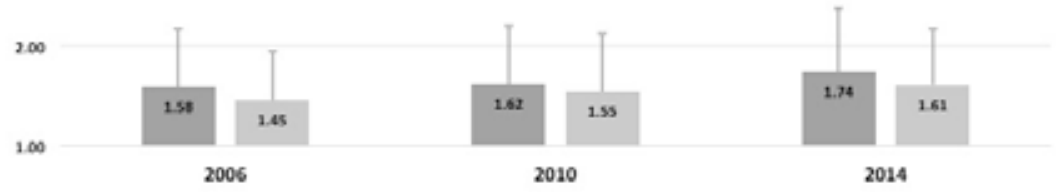

"Westschmeiz und Tesiis "Deutschichweis

Abbildung 4: Trend somatische Beschwerden nach Region 


\section{Zusammenfassung, Diskussion und Schlussfolgerungen}

Das Ziel des vorliegenden Beitrages ist es, die Verbreitung der negativen Beanspruchungsfolgen von Schweizer Lehrpersonen erstmals in einem nationalen Vergleich und zwischen drei Erhebungsjahren zu beschreiben.

In der Erhebung 2014 ist die negative Beanspruchung in Form der Arbeitsüberforderung im Durchschnitt auf mittlerem Niveau angesiedelt. Immerhin jede fünfte Lehrperson findet, dass man im Lehrberuf ständig überfordert wird und der Zeitdruck zu gross ist. Rund zwei Drittel der befragten Personen leiden unter Müdigkeit, ein Viertel berichtet von regelmässigen Schlafstörungen. Die Arbeitsunzufriedenheit ist trotzdem vergleichsweise tief, über 90\% der Lehrkräfte macht der Beruf Spass, lediglich 13.6\% haben sich schon mal ernsthaft überlegt, aus dem Beruf auszusteigen. Grundsätzlich lässt sich deshalb festhalten, dass die Schweizer Lehrkräfte der 5. - 9. Schulstufe trotz hoher Beanspruchung zufrieden sind in ihrem Beruf. Dies bestätigt Befunde aus anderen Studien (u.a. Bieri, 2006; Schult et al., 2015). Dass Lehrpersonen negativ beansprucht, aber trotzdem zufrieden sind, lässt sich mit den Merkmalen des Berufes erklären: Die hohe Autonomie, Flexibilität und Verantwortung führen einerseits zu Zufriedenheit (vgl. van Dick \& Stegmann, 2013), andererseits führt diese Konstellation zur Gefahr, dass die eigene Gesundheit vernachlässigt wird (vgl. Baeriswyl, Krause \& Kunz Heim, 2014).

In allen untersuchten Dimensionen findet sich ein regionaler Effekt, die Westschweizer und Tessiner Lehrkräfte sind stärker negativ beansprucht als die Deutschschweizer Lehrpersonen. Dieser Effekt wurde bereits im Erhebungsjahr 2002 festgestellt (Delgrande Jordan et al., 2005). Zusätzlich zeigen sich in der Westschweiz und dem Tessin mehr geschlechtstypische Unterschiede: Die Frauen fühlen sich stärker überfordert von der Arbeit als die Männer. In der Deutschschweiz findet sich dieser Unterschied nicht, was die Befunde von Keller-Schneider (2010) bestätigt. Bezüglich depressiven und somatischen Beschwerden findet sich in der ganzen Schweiz ein deutlicher Geschlechtsunterschied zuungunsten der Frauen. Dies bestätigt Befunde zu Erschöpfung und Burnout (Nübling et al., 2012; Schaufeli \& Enzmann, 1998).

Die Erwartung, dass sich die national bedeutsamen Schulreformen seit 2006 negativ auf das Befinden aller Schweizer Lehrkräfte der 5. - 9. Schulstufe ausgewirkt haben, bestätigt sich nur beschränkt in dieser Trendanalyse. Diese Trends müssen regional betrachtet werden:

In der Deutschschweiz bleibt das Ausmass der Arbeitsüberforderung und der depressiven Beschwerden stabil, die Arbeitsunzufriedenheit sinkt, die somatischen Beschwerden nehmen leicht zu. Die Trendanalyse ergibt diesbezüglich ein widersprüchliches Bild, insgesamt kann man aber sagen, dass sich die Anforderungen trotz der zahlreichen eingangs beschriebenen Reformen in der Deutschschweiz nicht verschärfend auf die negative Beanspruchung der Lehrkräfte 
ausgewirkt haben - dies obwohl Reformen in zahlreichen Studien als ein Hauptbelastungsfaktor genannt werden (Albisser et al., 2009; Nido, 2012; Trachsler et al., 2007). Dies bestätigt den Befund von Trachsler et al. (2007), die im Kanton Thurgau ebenfalls keine Zunahme der negativen Beanspruchung während einer Reform nachweisen konnten. Für die französisch- und italienischsprachige Schweiz zeigt sich ein leicht anderes Bild: Die Arbeitsüberforderung sinkt im Trend zwischen 2006 und 2010, nimmt danach jedoch deutlich zu, die somatischen Beschwerden steigen kontinuierlich. Die Arbeitsunzufriedenheit und die depressiven Beschwerden sind bei allen drei Erhebungsjahren stabil hoch. Für diese Regionen kann zwischen 2010 und 2014 eine Verschlechterung der Beanspruchungssituation festgestellt werden. Ob und wie dies zusammenhängt mit der flächendeckenden Implementation des «plan d'études romand» in der Westschweiz in diesem Zeitraum, kann mit den vorliegenden Analysen nicht abschliessend geklärt werden.

Reformen führen insbesondere dann zu Stresssymptomen, wenn «die Reform ungeliebt ist oder von vornherein als aussichtslos gilt» (Oelkers, 2000, S. 7), oder wenn die Umsetzung der Reform in der Praxis schwierig ist und nicht genügend Abwehrmechanismen bei den Lehrkräften vorhanden sind, mit diesem Ungenügen umzugehen (ebd.). Damit sind zwei wichtige Faktoren angesprochen, welche den Belastungs-Beanspruchungsprozess beeinflussen: Einerseits die organisatorischen Rahmenbedingungen und andererseits die individuellen Dispositionen der einzelnen Lehrkräfte. Nido (2012) wies nach, dass ein verkraftbares Mass an Reformprojekten, von deren Nutzen Lehrkräfte überzeugt sind, positiv mit dem Engagement von Lehrkräften zusammenhängt. Innovationen können eine positive Herausforderung sein und eine Chance für die professionelle Entwicklung, wenn die Umsetzung von Reformprojekten mit Hilfe von nachvollziehbaren Priorisierungen geordnet erfolgt (Oelkers, 2000). Das Sinken der Arbeitsunzufriedenheit in der Deutschschweiz könnte als Hinweis gedeutet werden, dass die Reformen der vergangenen Jahre positive Effekte auf das Lehrpersonal hatten. Welche Faktoren in der französisch- und italienischsprachigen Schweiz zur Verschlechterung der Situation geführt haben, kann zum jetzigen Zeitpunkt nicht festgestellt werden. Weiterführende Analysen zu den wahrgenommenen sozialen und personalen Ressourcen zwischen 2010 und 2014 und deren Zusammenhang mit der negativen und positiven Beanspruchung sind in Planung.

Die vorliegenden Daten erlauben einen Überblick über die Entwicklung der Beanspruchungssituation von Schweizer Lehrpersonen über einen längeren Zeitraum. Der Nachteil der Daten ist, dass Kohorten- und soziohistorische Unterschiede in den Stichproben nicht ausgeschlossen werden können. Eine längsschnittliche Untersuchung von verschiedenen Kohorten über den gleichen Zeitraum hätte sicherlich mehr Aussagekraft. Eine weitere Einschränkung ergibt sich durch den Abstand von vier Jahren zwischen den Erhebungen, wodurch kurzfristige Entwicklungen nicht erfasst werden. Die Querschnittstruktur 
verhinderte zudem die Kontrolle von Ausfällen - es wäre denkbar, dass stark beanspruchte Lehrkräfte den Beruf verlassen haben (Herzog, Herzog, Brunner \& Müller, 2007). Die beschränkte Anzahl vorhandener Einflussfaktoren auf der personalen und organisationalen Ebene haben zudem differenzierende Analysen in dieser Trendanalyse limitiert. Die vier Skalen und Indizes, die seit 2002 eingesetzt wurden und die Grundlage für diese Trendanalyse darstellen, sind nicht alle gleich geeignet, um negative Beanspruchung zu messen. Insbesondere für die Messung von depressiven Symptomen hätte es etabliertere Skalen gegeben.

In unserer Stichprobe sind Lehrpersonen mit einem Teilzeitpensum untervertreten. Verschiedene Studien zeigten, dass Lehrpersonen mit einem Teilzeitpensum höhere Beanspruchungen berichten, einerseits, weil sie stärker belastet sind durch Reformen, weniger Erholungsmöglichkeiten während der Pausen haben und Konferenzen und Sitzungen eher als Belastungen wahrnehmen (Kunz Heim, Sandmeier \& Krause, 2014a), andererseits weil sie weniger Unterstützung durch Schülerschaft und Eltern wahrnehmen und oft unter einer Mehrfachbelastung durch Erwerbs- und Familienarbeit leiden (Herzog, 2007). Inwiefern der Befund, dass die nationalen Bildungsreformen der vergangenen Jahre insgesamt nicht zu einer Zunahme in der negativen Beanspruchung geführt haben, auch auf die Teilzeitkräfte zutrifft, kann mit den vorliegenden Daten nicht beantwortet werden.

Um die Auswirkungen von Schulreformen auf die Belastung und Beanspruchung der Lehrkräfte detaillierter untersuchen zu können, sollten systematischer Daten erhoben werden, die Einflussfaktoren auf der personalen und organisationalen Ebene wenn möglich längsschnittlich feststellen, um individuelle Veränderungen und kausale Zusammenhänge feststellen zu können (Albisser et al., 2009). Die anstehende Implementation des Lehrplans 21 in verschiedenen Deutschschweizer Kantonen bietet die Gelegenheit, nicht nur die Umsetzung und die Effektivität der Reform zu evaluieren, sondern auch deren Auswirkungen auf die positive und negative Beanspruchung von Lehrpersonen zu erfassen. Dies könnte wichtige Hinweise liefern zur Frage, welche Faktoren auf der kontextuellen und der individuellen Ebene es den Lehrkräften erleichtert, die Massnahmen erfolgreich im Schulalltag umzusetzen, ohne dass ihre Gesundheit beeinträchtig wird (siehe auch Krause, Dorsemagen, \& Baeriswyl, 2013; Krause \& Dorsemagen, 2011).

\section{Anmerkungen}

1 Wir danken Dr. Marina Delgrande und M. Sc. Yvonne Eichenberger, Sucht Schweiz, und Prof. Dr. Silvio Herzog, Pädagogische Hochschule Schwyz, für die differenzierten Rückmeldungen zu diesem Beitrag.

2 Health Behaviour in School-Aged Children [HBSC], internationale Studie der Weltgesundheitsorganisation WHO zum Gesundheitsverhalten von Schülerinnen und Schülern zwischen 11 und 15 Jahren.

3 Windlin, Kuntsche \& Delgrande Jordan (2011). 
4 Für die Untersuchungsjahre 2010 und 2014 wurde eine Kooperation zwischen Sucht Schweiz, der Pädagogischen Hochschule FHNW und der Hochschule für Angewandte Psychologie FHNW eingegangen für die Fragebogenentwicklung und die Auswertung der Daten der Lehrerbefragung. Die Analysen wurden vom Schweizer Nationalfonds (Projektnr. 13DPD3_126807) finanziert.

5 Der Vergleich basiert auf den Daten von 2010 und 2014, in denen das gesamte unterrichtete Pensum erhoben worden ist. 2006 wurde lediglich das unterrichtete Pensum in der Klasse der HBSC-Erhebung erfragt.

6 Das Instrument von Enzmann und Kleiber enthält eine dritte Dimension (Kontrollerleben), die im Rahmen der vorliegenden Studie nicht zur Anwendung kam.

7 Das Antwortformat umfasst fünf Stufen $(5=$ etwa täglich, $4=$ mehrmals pro Woche, 3 = ungefähr einmal pro Woche, 2 = ungefähr einmal pro Monat und 1 = selten oder nie). Für die Analysen wurden die 5 Items zur Depressivität und die 4 Items zu somatischen Beschwerden summiert und durch die Anzahl einbezogener Items dividiert.

8 Antwortkategorie "trifft überwiegend zu», bzw. «trifft völlig zu».

9 Antwortkategorien «ungefähr einmal pro Woche», «mehrmals pro Woche» oder «etwa täglich».

\section{Literatur}

Albisser, S., Kirchhoff, E. \& Albisser, E. (2009). Berufsmotivation und Selbstregulation: Überfachliche Kompetenzentwicklung und Belastungserleben von Studierenden, berufseinsteigenden und erfahrenen Lehrpersonen. Unterrichtswissenschaft, 37, (3), 262 $-288$.

Baeriswyl, S., Krause, A. \& Kunz Heim, D. (2014). Arbeitsbelastungen, Selbstgefährdung und Gesundheit bei Lehrpersonen - eine Erweiterung des Job Demands-Resources Modells. Empirische Pädagogik, 28, (2), 128 - 146.

Bieri, T. (2006). Lehrpersonen: hoch belastet und trotzdem zufrieden? Bern: Haupt.

CIIP. (2015). Rapport annuel CIIP 2014. Neuchâtel: Conférence intercantonale de l'instruction publique de la Suisse Romande et du Tessin. Consulté le 3 janvier, 2017 dans http://www.ciip.ch/documents/showFile.asp?ID=7183

Criblez, L., Müller, B. \& Oelkers, J. (2011). Die Volksschule zwischen Innovationsdruck und Reformkritik. Zürich: Verlag Neue Zürcher Zeitung.

D-EDK. (2015). Schlussberichtzum Projekt Lehrplan 21. Luzern: Deutschschweizer Erziehungsdirektorenkonferenz. Luzern: D-EDK Geschäftsstelle. Zugriff am 13.10.2015 unter https:// www.lehrplan.ch/sites/default/files/Schlussbericht\%20Projekt\%20Lehrplan\%2021_ 2015-06-18.pdf

Delgrande Jordan, M., Kuntsche, E. N. \& Sidler, J. (2005). Arbeitsüberforderung und -unzufriedenheit von Lehrpersonen in der Schweiz. Zusammenhänge mit Depressivität und somatischen Beschwerden. Schweizerische Zeitschrift für Bildungswissenschaften, 27, (1), $123-139$.

Dick, R. van \& Stegmann, S. (2013). Belastung, Beanspruchung und Stress im Lehrerberuf - Theorien und Modelle. In M. Rothland (Ed.), Belastung und Beanspruchung im Lehrerberuf. Modelle, Befunde, Interventionen (S. 41-59). Wiesbaden: VS Verlag.

EDK. (2007). Interkantonale Vereinbarung über die Zusammenarbeit im Bereich der Sonderpädagogik. Bern: Schweizer Konferenz der kantonalen Erziehungsdirektoren. Zugriff am 13.10.2015 unter http://www.edudoc.ch/static/web/arbeiten/sonderpaed/konkordat_d. pdf

EDK. (2015). Bilanz 2015. Harmonisierung der verfassungsmässigen Eckwerte (Art. 62 Abs. 4 $B V)$ für den Bereich der obligatorischen Schule. Bern: Schweizer Konferenz der kantonalen Erziehungsdirektoren. Zugriff am 13.10.2015 unter http://edudoc.ch/record/117954/ files/pb_bilanz2015_d.pdf 
Enzmann, D. \& Kleiber, D. (1989). Helfer-Leiden. Stress und Burnout in psychosozialen Berufen. Heidelberg: Asanger.

Forneck, H.J. \& Schriever, F. (2001). Die individualisierte Profession. Belastungen im Lehrberuf. Bern: h.e.p. Verlag.

Grunder, H.U. \& Bieri, T. (1995). Zufrieden in der Schule? Zufrieden mit der Schule?: Berufszufriedenheit und Kündigungsgründe von Lehrkräften. Bern: Haupt.

Hacker, W. \& Reinhold, S. (1999). Beanspruchungsscreening bei Humandienstleistungen: BHD-System. Frankfurt am Main: Harcourt Test Services GmbH.

Hangartner, J., Hofer, K. \& Freisler, D. (2012). Belastungserfahrungen von Lehrpersonen im Kontext schulischer Reformen. vpod bildungspolitik, 176, 12-14.

Hakanen, J. J., Bakker, A. B. \& Schaufeli, W. B. (2006). Burnout and work engagement among teachers. Journal of School Psychology, 43, 495-513.

Herzog, S. (2007). Beanspruchung und Bewältigung im Lehrerberuf. Eine salutogenetische und biografische Untersuchung im Kontext unterschiedlicher Karriereverläufe. Münster: Waxmann.

Herzog, W., Herzog, S., Brunner, A. \& Müller, H. P. (2007). Einmal Lehrer, immer Lehrer? Eine vergleichende Untersuchung der Berufskarrieren von (ehemaligen) Lehrpersonen. Bern: Haupt.

Keller-Schneider, M. (2010). Entwicklungsaufgaben im Berufseinstieg von Lehrpersonen. Beanspruchung durch berufliche Herausforderungen im Zusammenhang mit Kontext- und Persönlichkeitsmerkmalen. Münster: Waxmann.

Klusmann, U., Kunter, M., Trautwein, U. \& Baumert, J. (2006). Lehrerbelastung und Unterrichtsqualität aus der Perspektive von Lehrenden und Lernenden. Zeitschrift für Pädagogische Psychologie, 20, (3), 161 - 173.

Krause, A. \& Dorsemagen, C. (2011). Gesundheitsförderung für Lehrerinnen und Lehrer. In E. Bamberg, A. Ducki, \& A.-M. Metz (Hrsg.), Gesundheitsförderung und Gesundheitsmanagement in der Arbeitswelt (S. 139-157). Göttingen: Hogrefe.

Krause, A., Dorsemagen, C. \& Baeriswyl, S. (2013). Zur Arbeitssituation von Lehrerinnen und Lehrern: Ein Einstieg in die Lehrerbelastungs- und -gesundheitsforschung. In M. Rothland (Hrsg.), Belastung und Beanspruchung im Lehrerberuf(S. 58 - 80). Wiesbaden: Springer.

Kristensen,T.S., Borritz, M., Villadsen, E. \& Christensen, K.B. (2005). The Copenhagen Burnout Inventory: A new tool for the assessment of burnout. Work \& Stress, 19, (3), 192-207.

Kunz Heim, D., Sandmeier, A. \& Krause, A. (2014a). Negative Beanspruchungsfolgen bei Schweizer Lehrpersonen. Beiträge zur Lehrerbildung, 32, (2), 280 - 295.

Kunz Heim, D., Sandmeier, A. \& Krause, A. (2014b). Effekt von arbeitsbedingten und personalen Ressourcen auf das Arbeitsengagement und das Engagement für Schulentwicklung bei Lehrpersonen. Empirische Pädagogik, 28, (1), 147 - 170.

Landert, C. (2014). Die Berufszufriedenheit der Deutschschweizer Lehrerinnen und Lehrer. Bericht zur vierten Studie des Dachverbandes Lehrerinnen und Lehrer Schweiz (LCH). Zürich: Landert Brägger Partner.

Landert, C. \& Brägger, M. (2009). LCH Arbeitszeiterhebung 2009. Zürich: Landert Partner.

Lehr, D., Hillert, A. \& Keller, S. (2013). What can balance the effort? Associations between effort-reward imbalance, overcommitment, and affective disorders in German teachers. International journal of occupational and environmental health, 15, (4), 374-384.

Maslach, C. \& Jackson, S. (1981). Maslach Burnout Inventory. Research Edition. Palo Alto: Consulting Psychologists Press.

Moser Opitz, E. (2011). Integrative Schulung. In L. Criblez, B. Müller \& J. Oelkers (Hrsg.), Die Volksschule zwischen Innovationsdruck und Reformkritik (S. 140 - 150). Zürich: Verlag Neue Zürcher Zeitung.

Nido, M. (2012). Das Engagement von Lehrpersonen: gesund und arbeitsfreudig oder ausgebrannt 
und reformmüde?Verschiedene Engagement-Typen und ihr Zusammenhang mit Arbeitsbedingungen, Personen- und Schulmerkmalen. Lengerich: Pabst Science Publishers.

Nido, M., Trachsler, E., Ackermann, K., Brüggen, S. \& Ulich, E. (2008). Arbeitsbedingungen, Belastungen und Ressourcen von Lehrpersonen und Schulleitungen im Kanton Aargau. Zürich: Institut für Arbeitsforschung und Organisationsberatung. Zugriff am 03.01.2017 unter http://edudoc.ch/record/29732/files/08-11-arbeitszeitstudie_bericht.pdf

Nübling, M., Vomstein, M., Haug, A., Nübling, T., Stössel, U., Hasselhorn, H-M. et al. (2012). Befragung zu psychosozialen Faktoren am Arbeitsplatz. Personenbezogene Gefährdungsbeurteilung an öffentlichen Schulen in Baden-Württemberg. Freiburg: Freiburger Forschungsstelle Arbeits- und Sozialmedizin.

Oelkers, J. (2000). Stress und Last im Lehrerberuf heute. Vortrag gehalten am 11. September 2000 im Lehrerverein Chur. Zugriff am 29.08.2015 unter http://www.geser.net/gesleh/ fs09arb/oelkers.pdf

Rothland, Martin (2013). Belastung und Beanspruchung im Lehrerberuf. 2. vollständig überarbeitete Auflage. Wiesbaden: Springer.

Rudow, B. (1994). Die Arbeit des Lehrers: Zur Psychologie der Lehrertätigkeit, Lehrerbelastung und Lehrergesundheit. Bern: Huber.

Rudow, B. (2000). Der Arbeits-und Gesundheitsschutz im Lehrerberuf: Gefährdungsbeurteilung der Arbeit von Lehrerinnen und Lehrern. Stuttgart: Süddt. Pädagogischer Verlag.

Schaarschmidt, U. \& Fischer, A. W. (1997). AVEM-ein diagnostisches Instrument zur Differenzierung von Typen gesundheitsrelevanten Verhaltens und Erlebens gegenüber der Arbeit. Zeitschrift für Differentielle und Diagnostische Psychologie, 18, (3), 151 - 163.

Schaarschmidt, U. \& Kieschke, U. (2013). Beanspruchungsmuster im Lehrerberuf. In M. Rothland (Hrsg.), Belastung und Beanspruchung im Lehrerberuf(S. 81 - 98). Wiesbaden: Springer VS.

Schaufeli, W. \& Enzmann, D. (1998). The burnout companion to study and practice: A critical analysis. CRC press.

Schult, J., Münzer-Schrobildgen, M. \& Sparfeldt, J. R. (2015). Belastet, aber hochzufrieden? Zeitschrift für Gesundheitspsychologie, 22, (2), 61 - 67.

Siegrist, J. (1996). Adverse health effects of high effort-low reward conditions. Journal of Occupational Health Psychology, 1, (1), 27-41.

Trachsler, E., Brüggen, S., Nido, M., Ulich, E., Inversini, S., Wülser, M. \& Herms, I. (2006). Arbeitsbedingungen, Belastungen und Ressourcen in der Thurgauer Volksschule-Teilstudie Lehrkräfte. Frauenfeld: PHTG, Forschung.

Ulich, E. \& Wülser, M. (2015). Gesundheitsmanagement in Unternehmen. Arbeitspsychologische Perspektiven. 6. erw. Aufl. Wiesbaden: Gabler.

Ulich, E., Inversini, S. \& Wülser, M. (2002). Arbeitsbedingungen, Belastungen und Ressourcen der Lehrkräfte des Kantons Basel-Stadt. Zürich: Institut für Arbeitsforschung und Organisationsberatung.

Windlin, B., Kuntsche, E. \& Delgrande Jordan, M. (2011). Arbeitsüberforderung und-unzufriedenheit von Lehrpersonen in der Schweiz. National repräsentative Ergebnisse demografischer, klassen-und schulbezogener Faktoren. Schweizerische Zeitschrift für Bildungswissenschaften, 33, (1), 125 - 144.

\section{Schlagworte: Gesundheit von Lehrpersonen, Belastung, Bildungsreformen}




\section{Sollicitations à l'égard des enseignant-es en Suisse. Tendances de 2006 à 2014}

\section{Résumé}

Cet article examine si et dans quelle mesure l'ampleur des sollicitations à l'égard des enseignant-es suisses de $5^{\mathrm{e}}$ à $9^{\mathrm{e}}$ année scolaire (7e à $11 \mathrm{e}$ HarmoS) a évolué au cours des dix dernières années en Suisse. Sur la base des données d'études transversales répétées au plan national, l'évolution entre 2006 et 2014 a pu être décrite pour la première fois: globalement, l'ampleur des sollicitations est restée relativement stable entre les années, mais on observe des évolutions différentes selon les régions. L'enquête montre également que les enseignant-es restent satisfait-es de leur profession malgré des exigences de plus en plus élevées. Les résultats sont discutés en relation avec les réformes scolaires significatives qui ont eu lieu en Suisse durant cette période.

Mots-clés: Enseignants, satisfaction professionnelle, charge de travail, réforme scolaire

\section{Sollecitazioni e stress degli insegnanti in Svizzera. Evoluzione dal 2006 al 2014}

\section{Riassunto}

Questo articolo esamina se, e in quale misura, le sollecitazioni degli insegnanti svizzeri di $5^{\circ}$ a $9^{\circ}$ anno scolastico $\left(7^{\circ}\right.$ a $\left.11^{\circ} \mathrm{HarmoS}\right)$ sono evolute nel corso degli ultimi 10 anni. Sulla base dei risultati di studi trasversali ripetuti a livello nazionale, levoluzione tra 2006 e 2014 ha potuto essere descritta per la prima volta. Globalmente, il livello delle sollecitazioni è rimasto relativamente stabile negli anni, anche se si osservano delle evoluzioni differenti secondo la regione linguistica, e gli insegnanti sono soddisfatti della loro professione nonostante le esigenze in costante aumento. I risultati vengono discussi in relazione alle significative riforme scolastiche avvenute in Svizzera durante il periodo analizzato.

Parole chiave: Insegnanti, soddisfazione professionale, carico professionale, riforma scolastica 


\section{Subjective Stress and Strain of Swiss Teachers. Trends from 2006 to 2014}

\section{Summary}

This paper discusses the subjective stress and strain of Swiss teachers of 5th to 9th grade(rs) on three representative random samples in the years 2006, 2010 and 2014. The assumptions of increasing subjective strain and health complaints and decreasing job satisfaction were not supported: These dimensions remain stable over time or improve even slightly. At the same time the results indicate differences between men and women and between the different language regions. The results are discussed against the background of the educational reforms on the national level.

Keywords: Teachers, professional satisfaction, work stress, school reform 